\title{
Wind-shearing in gaseous protoplanetary disks
}

\author{
Hagai B. Perets ${ }^{1}$ and Ruth Murray-Clay ${ }^{1}$ \\ ${ }^{1}$ Harvard-Smithsonian Center for Astrophysics, 60 Garden st. Cambridge MA 02338, US \\ email: hperets@cfa.harvard.edu
}

\begin{abstract}
One of the first stages of planet formation is the growth of small planetesimals and their accumulation into large planetesimals and planetary embryos. This early stage occurs much before the dispersal of most of the gas from the protoplanetary disk. Due to their different aerodynamic properties, planetesimals of different sizes/shapes experience different drag forces from the gas at these stage. Such differential forces produce a wind-shearing effect between close by, different size planetesimals. For any two planetesimals, a wind-shearing radius can be considered, at which the differential acceleration due to the wind becomes greater than the mutual gravitational pull between the planetesimals. We find that the wind-shearing radius could be much smaller than the gravitational shearing radius by the Sun (the Hill radius), i.e. during the gas-phase of the disk wind-shearing could play a more important role than tidal perturbations by the Sun. Here we study the wind-shearing radii for planetesimal pairs of different sizes and compare it with gravitational shearing (drag force vs. gravitational tidal forces). We then discuss the role of wind-shearing for the stability and survival of binary planetesimals, and provide stability criteria for binary planetesimals embedded in a gaseous disk.
\end{abstract}

Keywords. planets and satellites: dynamical evolution and stability, planets and satellites: formation, planetary systems: protoplanetary disks

The interactions between planetesimals play an important role in the evolution of protoplanetary disks and planet formation (Lissauer 1993; Goldreich et al. 2004). Most of these interactions and the growth of planetesimals likely occur while the planetesimals are embedded in a gaseous disk. Here we focus on the close interaction between pairs of single planetesimals, i.e. planetesimal-planetesimal-gas interactions.

Planetesimals could vary in size and shape, and therefore have a wide range of aerodynamical properties, which would affect their interaction with the surrounding gas. In particular, planetesimals of different sizes/shapes experience different drag forces from the head wind they encounter in the gaseous disk. The difference between the forces acting on two, different size planetesimals, could effectively change their relative trajectories in respect to their unperturbed motion in the absence of gas (see also Ormel \& Klahr 2010). In particular, during an encounter between two different size planetesimals such differential forces result in a wind-shearing (WISH) effect, which could be even stronger than their gravitational interaction. For any two planetesimals, a WISH radius can be considered, at which the acceleration due to aerodynamical wind-shearing becomes greater than the mutual gravitational pull between them. Binary planetesimals can not survive with separation larger than the WISH radius, even if they are gravitationally bound. These issues are discussed in detail in Perets \& Murray-Clay (2011).

The differential acceleration between two planetesimal of radii $r_{b}$ and $r_{s}$, due to the wind-shearing effect is given by $\Delta a=\left|F_{D}\left(r_{b}\right) / m_{b}-F_{D}\left(r_{s}\right) / m_{s}\right|$, where $F_{D}(r)$ is the drag force affecting a planetesimal of radius $r$.

For small distances, at which the environmental conditions are approximately the same, the differential WISH between any two planetesimals is independent of the 


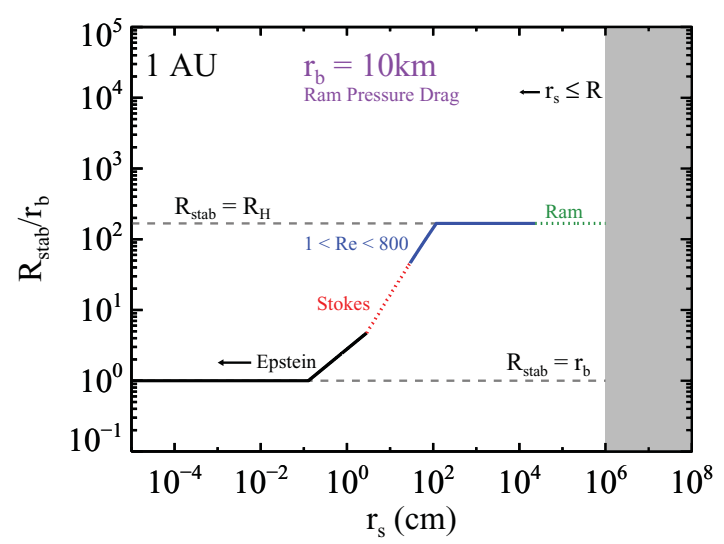

Figure 1. The WISH radius of a $10 \mathrm{~km}$ planetesimal and a planetesimal of size $r_{s}$, as a function of $r_{s}$ at $1 \mathrm{AU}$ from the star. The physical size and the Hill radii of the large size planetesimal (lower and upper dashed lines, respectively), are also shown. Also shown are the appropriate drag regimes, which depend on the Reynolds number Re.

distance between them. However, similar to the the Hill radius which arises from the tidal graviataional shearing from the Sun, we can define an important relevant distance scale; the wind-shearing radius. We define this radius as the distance between two planetesimals for which the differential WISH acceleration between them equals their mutual gravitational pull. Beyond this limiting radius even two planetesimals which are gravitationally bound (in the absence of WISH) would be sheared apart by the wind.

We can now derive the WISH radius for any given pair of planetesimals, by equating $\Delta a_{W S}$ with the gravitational acceleration $a_{g r a v}=G M / r_{W S}^{2}$ at the WISH radius $\left(r_{W S}\right)$.

The WISH radius can be found for any two planetesimals of arbitrary size, as illustrated in fig. 1. This figure shows the calculated WISH radius as a function of the small planetesimal size. The transition between different drag regimes can be seen in this figure.

Binary planetesimals could be strongly affected by gas drag. In a gas free environment, binary planetesimals are stable as long as their separation is smaller than the Hill radius, whereas wider binaries are destabilized and disrupted by the tidal gravitational shearing from the Sun. However, when gas drag is taken into account, the Hill radius stability limit should be replaced by the WISH radius (as long as $r_{W S}<r_{\text {Hill }}$; binaries wider than the Hill radius are always unstable), i.e. we can formulate a stability criteria for binaries embedded in gas: $a_{b i n} \leqslant \min \left(r_{H i l l}, r_{W S}\right)$. In Fig. 1 the WISH radius represents the limiting separations of binary planetesimals $\left(R_{\text {stab }}\right)$ or small satellites embedded in a gaseous environment at ahich bound binary planetesimals can exist. The appropariate phase space is delimited by the physical radii, the WISH radii, and the Hill radii.

\section{References}

Goldreich, P., Lithwick, Y., \& Sari, R. 2004, ARAA, 42, 549

Lissauer, J. J. 1993, ARAA, 31, 129

Ormel, C. W. \& Klahr, H. H. 2010, $A \& A$, 520, A43

Perets, H. B. \& Murray-Clay, R. A. 2011, ApJ, 733, id.56 